\title{
Morphological aspects of the euphonic voice
}

\author{
Bożena Kosztyła-Hojna ${ }^{1}$, Anna Andrzejewska ${ }^{2}$, Diana Moskal', Marek Rogowski ${ }^{3}$, \\ Dawid Falkowski ${ }^{1}$, Joanna Kasperuk ${ }^{1}$ \\ ${ }^{1}$ Department of Clinical Phonoaudiology and Logopedics, Medical University of Bialystok, Poland \\ ${ }^{2}$ Department of Medical Pathomorphology, Medical University of Bialystok, Poland \\ ${ }^{3}$ Department of Otolaryngology, Medical University of Bialystok, Poland
}

\begin{abstract}
The high quality of a euphonic voice is the result of complex interactions between many organs and systems. Vibrating vocal folds play a crucial role in this process. Their physiological motion is conditioned by the presence of the layered structure of laryngeal mucosa. In this study, we assessed the degree of dysphonia according to the Union of European Phoniatrics (UEP) scale. Videoendoscopy (VLS) and videostroboscopic (VLSS) examination of the larynx was used to visualize the vibration of the vocal folds. Morphological assessment of the inter-membranous part of the vocal fold mucosa was carried out using material collected after surgical treatment $(60 \%)$ or obtained from autopsy $(40 \%)$. The samples were examined by light microscopy and transmission electron microscopy. In euphonic voices, $1^{\circ}$ of dysphonia (UEP) and the physiological endoscopic (VLS) and stroboscopic (VLSS) findings of vocal folds were registered. No morphological or ultramorphological changes were observed in the cells of the multilayered flat epithelium, basal membrane or in the stroma. Unchanged epithelial cells were situated on the basal membrane with folds. Moreover, numerous pericytes, vessels with multiplication of basal membranes, scanty collagenous fibers, plasmatic cells and lymphocytes were seen. Morphological changes with signs of atrophy and polypoid degeneration of the vocal fold mucosa were found in only $3(15 \%)$ patients. (Folia Histochemica et Cytobiologica 2011; Vol. 49, No. 1, pp. 72-79)
\end{abstract}

Key words: larynx, epithelial cells, pericytes, lamina propria, videolaryngostroboscopy, phonation, voice quality, polypoid degeneration

\section{Introduction}

The high quality of voice - euphonic voice — depends on the integral functioning of many systems and organs, the most important of which are the larynx and vibrating vocal folds [1]. An efficiently functioning vibrating mechanism causes the rhythmical opening and closing of the glottis, constituting the fundamental frequency $\left(\mathrm{F}_{0}\right)$. The correct functioning of the vocal folds is conditioned by their layered structure. The vocal folds

Correspondence address: B. Kosztyła-Hojna,

Department of Clinical Phonoaudiology and Logopedics,

Medical University of Bialystok

J. Kilińskiego Str. 1, 15-089 Bialystok, Poland;

tel.: (+ 48 85) 74856 52; fax: (+ 48 85) 74856 51;

e-mail: fono@umwb.edu.pl include a vocal muscle and mucosa which consists of epithelium and lamina propria [1]. For physiological phonation, the most important is the superficial layer of lamina propria, which is soft, prone to edema or bleeding, and causes the motion of the epithelium during the vibration of the vocal folds. The intermediate and deep layers are more fibrous and constitute the vocal ligament. The vocal muscle forms the main mass of the vocal folds. Pathological voice quality - dysphonia - may be the result of functional or organic pathology [2,3]. Organic changes may refer to the epithelium or lamina propria of the laryngeal mucosa. The phonatory organ and larynx have great compensatory capacities, allowing good voice quality even with coexisting pathology of the larynx.

The aim of this study was to provide a morphological assessment of the mucosa of the vocal folds in persons with euphonic voice. 


\section{Material and methods}

A group of 20 patients, 15 (75\%) male and $5(25 \%)$ female, was included in the study (Table 1). Material for morphological examination was obtained from 12 patients aged 39-45 years (average 41 years) with hypopharyngeal cancer without coexisting pathological changes of the vocal folds, who had been treated with total laryngectomy. Post-operative examination of this group of patients demonstrated the presence of squamous cell carcinoma (Table 2). In the remaining eight cases, the material for morphological examination was collected during autopsy. In the autopsy material, no neoplasmatic changes of the larynx or hypopharynx were registered. The material obtained from autopsy constituted the re-

Table 1. Clinical characteristics of the analyzed groups of patients

\begin{tabular}{|l|c|c|c|c|}
\hline \multirow{2}{*}{ Data } & \multicolumn{2}{|c|}{$\begin{array}{c}\text { Material after } \\
\text { surgery }\end{array}$} & \multicolumn{2}{c|}{$\begin{array}{c}\text { Autopsy } \\
\text { material }\end{array}$} \\
\cline { 2 - 5 } & Female & Male & Female & Male \\
\hline $\begin{array}{l}\text { Total number of } \\
\text { patients in the } \\
\text { group (\%) }\end{array}$ & \multicolumn{2}{|c|}{$12(60 \%)$} & \multicolumn{2}{|c|}{$8(40 \%)$} \\
\hline $\begin{array}{l}\text { Number of } \\
\text { patients (\%) }\end{array}$ & $2(17 \%)$ & $10(83 \%)$ & $3(37 \%)$ & $5(63 \%)$ \\
\hline Age span & $39-43$ & $40-45$ & $35-38$ & $40-45$ \\
\hline Average & 42 & 43 & 36 & 41 \\
\hline $\begin{array}{l}\text { Average } \\
\text { in the group }\end{array}$ & \multicolumn{2}{|c|}{41} & \multicolumn{2}{|c|}{} \\
\hline
\end{tabular}

Table 2. Histopathological changes and clinical characteristics of patients with hypopharyngeal cancer

\begin{tabular}{|c|c|c|}
\hline \multicolumn{2}{|l|}{ Data } & Material after surgery \\
\hline \multicolumn{2}{|c|}{$\begin{array}{l}\text { Hypopharyngeal cancer } \\
\text { (squamous cell carcinoma) }\end{array}$} & 12 \\
\hline \multicolumn{2}{|c|}{$\begin{array}{l}\text { Normal morphological } \\
\text { structure of vocal fold mucosa }\end{array}$} & 9 \\
\hline \multicolumn{2}{|c|}{ Atrophy of vocal fold mucosa } & 1 \\
\hline \multicolumn{2}{|l|}{$\begin{array}{l}\text { Polypoid degeneration } \\
\text { of vocal fold mucosa }\end{array}$} & 2 \\
\hline \multirow[t]{2}{*}{ Tumor $(\mathrm{T})$} & $\begin{array}{l}\mathrm{T}_{1} \\
(\%)\end{array}$ & $\begin{array}{c}9 \\
(75 \%)\end{array}$ \\
\hline & $\begin{array}{c}\mathrm{T}_{2} \\
(\%)\end{array}$ & $\begin{array}{c}3 \\
(25 \%)\end{array}$ \\
\hline Nodulus (N) & $\mathrm{N}_{0}$ & $100 \%$ \\
\hline Metastases (M) & $\mathrm{M}_{0}$ & $100 \%$ \\
\hline \multirow[t]{2}{*}{ Clinical advancement } & $\begin{array}{l}\mathrm{T}_{1} \mathrm{~N}_{0} \mathrm{M}_{0} \\
\text { (I) }(\%)\end{array}$ & $\begin{array}{c}9 \\
(75 \%)\end{array}$ \\
\hline & $\begin{array}{l}\mathrm{T}_{2} \mathrm{~N}_{0} \mathrm{M}_{0} \\
\text { (II) }(\%)\end{array}$ & $\begin{array}{c}3 \\
(25 \%)\end{array}$ \\
\hline
\end{tabular}

ference group for comparison with patients with hypopharyngeal cancer. The age range for the group sampled at autopsy was 35-45 years (average 38 years).

A subjective assessment of voice quality was carried out in patients with hypopharyngeal cancer before surgical treatment using the 6-degree scale of dysphonia of the Union of European Phoniatrics, where $1^{\circ}$ refers to physiological voice - euphonic, $2^{\circ}$ - hoarseness, $3^{\circ}$ - dysphonia of mild degree, $4^{\circ}-$ moderate degree of dysphonia, $5^{\circ}-$ severe degree of dysphonia and $6^{\circ}$ - aphonia or vicarious voice.

Endoscopic assessment was carried out using videoendoscopic (VLS) and videolaryngoscopic (VLSS) examination with the aid of a videolaryngoscope with 90-degree optics. Various parameters of stroboscopic assessment were used in the clinical diagnosis: mucosal wave, vibration amplitude, and phonatory closure of the glottis.

The material for morphological examination was obtained from the inter-membranous part of the vocal folds. Morphological assessment was carried out using light microscopy and transmission electron microscopy (TEM). Specimens of laryngeal mucosa used for morphological examination by light microscopy were fixed in $10 \%$ formalin solution and subsequently dehydrated in the ascending alcohol row. The material was mounted in paraffin blocks, dissected specimens were stained with hematoxylin and eosin $(\mathrm{H}+\mathrm{E})$, and evaluated using light microscopy.

The material for morphological examination by TEM was fixed in $2.5 \%$ glutaric aldehyde in kakodylate buffer, $\mathrm{pH}=7.4$ for four hours and postfixed in $1 \% \mathrm{OsO} 4$ for two hours and mounted in Epon 812. Half-thin specimens were stained with methylene blue and preliminarily evaluated using light microscopy. Ultra-thin specimens were obtained using an ultramicrotome (Reichert Ultracuts), contrasted with lead citrate and uranyl acetate and subsequently analyzed using a transmission electron microscope (Opton $900 \mathrm{PC}$ ).

\section{Results}

In the group of patients with hypopharyngeal cancer, the voice quality disorders of dysphonia were not registered from hearing. In 2 patients (17\%), a slight hoarseness after speaking for a long time was registered. The main symptom reported by all patients was a sore throat, especially during eating, and pain radiating to the ear was reported by $3(25 \%)$ patients. Voice analysis showed $1^{\circ}$ dysphonia in 10 $(83 \%)$ patients and $2^{\circ}$ dysphonia in the other $2(17 \%)$ patients.

Endoscopic assessment of the larynx revealed that in $11(92 \%)$ patients, the vocal folds were regular, 
smooth and moveable during respiration and phonation, with physiological phonatory closure of the glottis. In $1(8 \%)$ patient, lack of complete phonatory glottic closure and increased mass of the vocal folds were observed. In the stroboscopic assessment, 10 (83\%) patients demonstrated a regular vibration amplitude with the presence of a normal mucosal wave and physiological phonatory glottic closure. (Figure 1)

In $2(17 \%)$ patients, we found increased vibration amplitude and a limited mucosal wave and physiological phonatory closure of the glottis. In $1(8 \%)$ patient we observed a decreased vibration amplitude and limited mucosal wave with lack of phonatory glottic closure.

Morphological examination of the vocal fold mucosa obtained from the study group of 20 patients via light microscopy and staining with hematoxylin and $\operatorname{eosin}(\mathrm{H}+\mathrm{E})$ revealed multilayered flat epithelium alongside the edge of the vocal fold. Multistratified ciliated epithelium was registered in the area of the ventricle of the larynx, and above the glottis. The multilayered epithelium was without signs of keratosis. Moreover, we observed occasional infiltrations from plasmatic cells and lymphocytes under the epithelium (Figure 2).

The results obtained from TEM indicated the presence of multilayered flat epithelium with pronounced folds in the basal membrane. Furthermore, blood vessels with multiplications of the basal membrane (BM), a large number of pericytes $(\mathrm{P})$ and scanty collagenous fibers $(C)$ in the stroma were demonstrated (Figure 3 ).

No significant ultrastructural changes with the presence of intercellular spaces were found in epithelial cells (Figure 4).

In two cases, enlarged intercellular spaces of the epithelium and mild inflammation in the area of the epithelium and under the epithelium were observed. The basal membrane of the epithelium appeared to be slightly folded (Figure 5).

The ultramorphological findings revealed signs of vacuolar degeneration of the epithelial cells, the presence of collagenous fibers $(\mathrm{C})$ and fibroblasts $(\mathrm{F})$ in the stroma. Moreover, fibroblasts showed the traits of vacuolization (Figures 6,7 ).

In one case, scanty collagenous fibers $(\mathrm{C})$ and fibroblasts $(\mathrm{F})$ were registered in the stroma of mucosa. The basal membrane of the epithelium appeared to have small folds (Figure 8).

\section{Discussion}

The regular vibration of the vocal folds depends on the physiological layered structure of lamina propria of the vocal fold mucosa. The multilayered, flat epithelium, without signs of keratosis of the vocal fold mucosa, is situated on the basal membrane. In young people, the folds of the basal membrane are pronounced [2-5]. The lamina propria of the mucosa consists of a superficial layer, an intermediate layer and a deep layer [6].

From the point of view of function, layers of the vocal fold mucosa can be divided into three sections: the first comprises the epithelium with superficial layer forming a cover and giving shape to the vocal fold, and the intermediate and deep layers, which support the functions of transmission and movement of the vocal fold. The third section consists of the vocal muscle constituting the mass of the vocal fold. The superficial and deep layers create the fastening structure - the vocal ligament. The epithelium covering the vocal fold mucosa determines the proper shape of the vocal fold, which is evaluated using indirect laryngoscopy. The superficial layer is soft and prone to edema and bleeding, which is significant for the physiological vibration of the vocal fold $[1,3]$. This complicated layered structure of the vocal fold enables vibrations of the vocal folds during phonation in three planes: horizontal, vertical and sagittal.

The fundamental frequency $\left(\mathrm{F}_{0}\right)$, which has an impact on voice quality, is constituted on the vocal folds. The physiological motion and vocal fold vibration determine the physiological voice, which is called euphonic [3, 7]. In the analyzed material, in $83 \%$ of cases the voice was described from hearing as euphonic and classified as $1^{\circ}$ according to the UEP scale. Sore throat and earache were the major complaints reported by patients. The aforementioned symptoms were caused by the hypopharyngeal localization of the tumor.

Morphological changes occurring in the mucosa and disturbing the physiological - layered structure, lead to the pathological vibration of the vocal folds. This was the cause of the voice quality disorder known as dysphonia. Morphological changes occurring in the mucosa have been described in patients with the clinical form of edema and atrophy of the larynx and in patients in the senile age [2, 8-15]. Atrophy of vocal fold mucosa, a process that occurs with age, results in disorders of voice quality called presbyphonia. Kosztyła-Hojna, Sato et al. [2,12] have described thinning of the paraepidermoid epithelium and partly multistratified epithelium with infiltrations from plasmatic cells and lymphocytes in patients over the age of 65. In ultramorphological examinations, a large number of collagenous fibers (C), inflammatory cells and fibroblasts (F) with signs of vacuolization and small folds occurring in the basal membrane have been observed. 


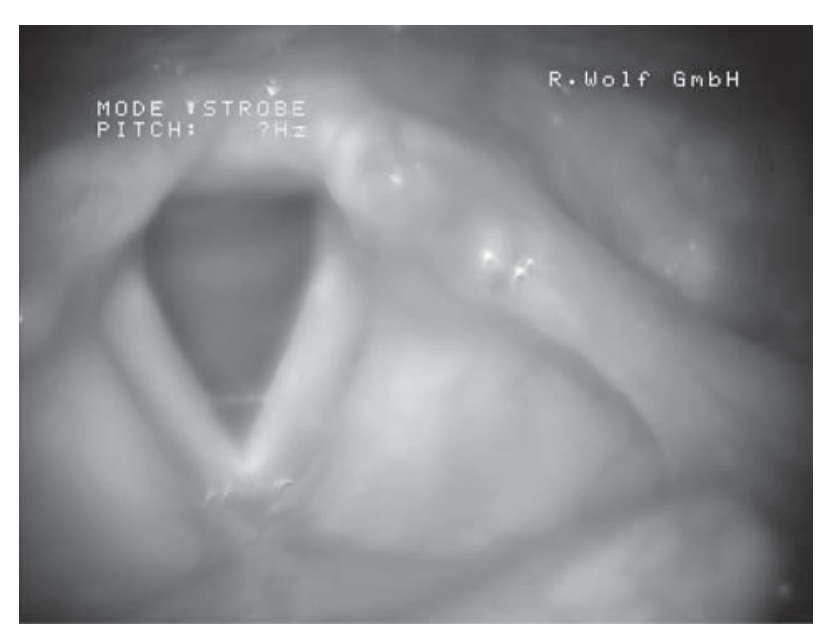

Figure 1. Endoscopic finding of larynx in patient with euphonic voice

In the analyzed material, only one patient demonstrated findings similar to the aforementioned morphological changes (Figure 7). Laryngeal edemas are often seen in older female patients, but atrophy is seldom observed. Laryngeal edemas are characterized by keratosis of the paraepidermoid epithelium with enlarged blood vessels and infiltrations from plasmatic cells and lymphocytes [2]. The intensified destruction of epithelial cells with vacuolization, small folds of the basal membrane, the occurrence of fibroblasts $(\mathrm{F})$ with enlarged cisterns of the granular endoplasmatic reticulum in branches and an increased number of collagenous fibers (C) observed in ultramorphological assessment suggest the presence of edematous changes of the vocal fold mucosa. In the analyzed material, only two patients demonstrated enlarged intercellular spaces of the epithelium with traits of vacuolar degeneration together with a small number of collagenous fibers and fibroblasts in the stroma with signs of vacuolization.

In the morphological material obtained from the remaining 17 persons, multilayered flat epithelium with intercellular spaces, basal membrane with folds, numerous blood vessels with multiplication of basal membrane and a large number of pericytes and scanty collagenous fibers were registered. According to many authors, a large number of pericytes are found in the mucosa of vibrating structures in neonates and young people [16-19]. The function of the pericytes is to support and protect the blood vessel walls in vibrating vocal folds.

The mechanism of phonation and voice quality in patients with registered pathology of the larynx depends, to a large extent, on the compensatory capacities of the larynx and the ability to work with the voice [3]. The creation of physiological voice euphonic voice - is an art and can occur even with coexisting pathology of the laryngeal mucosa.

\section{Conclusions}

1. The euphonic voice is conditioned by the normal morphological structure of the vibrating vocal fold mucosa.

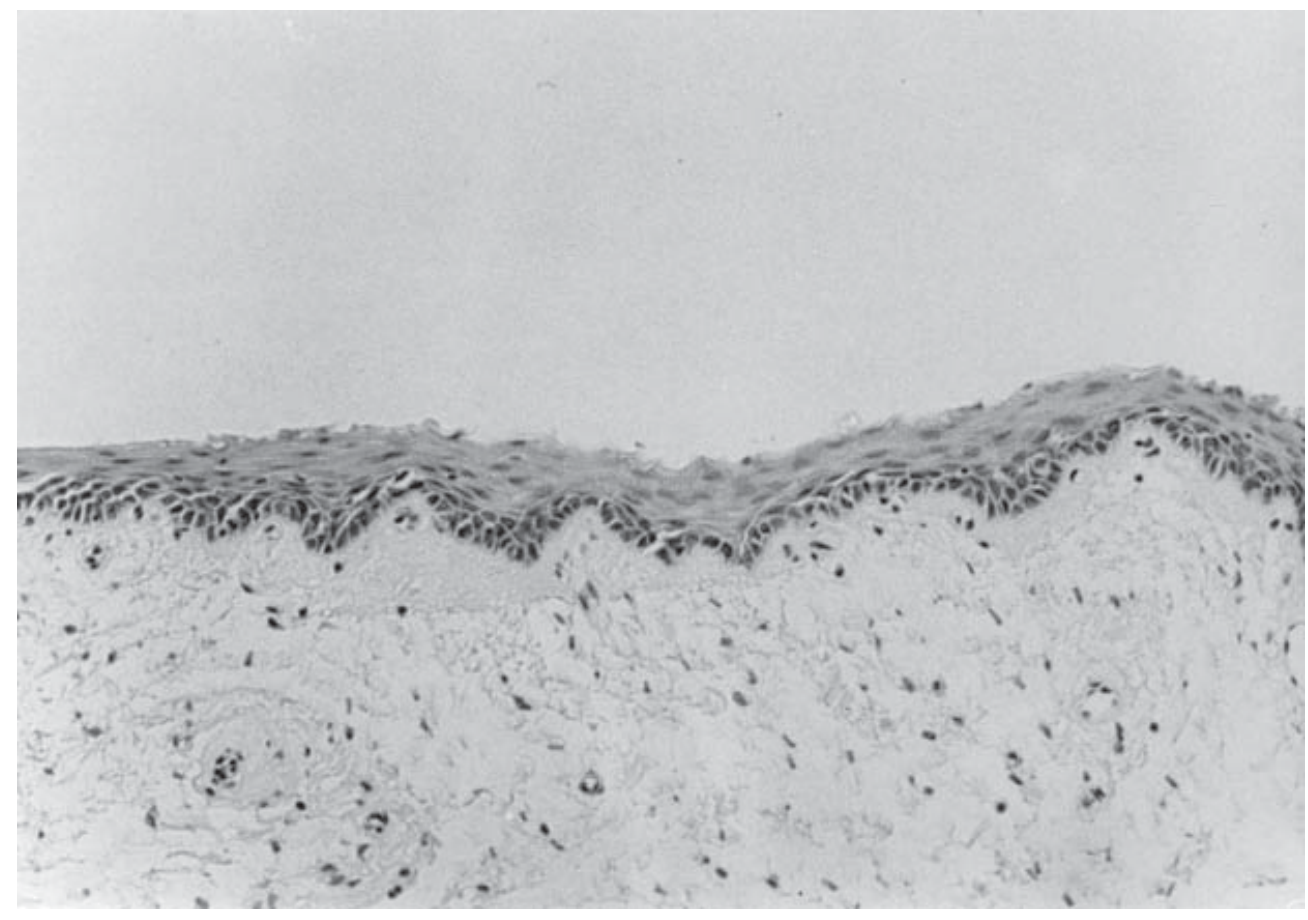

Figure 2. Vocal fold mucosa $(H+E$, magnification $\times 300)$ 


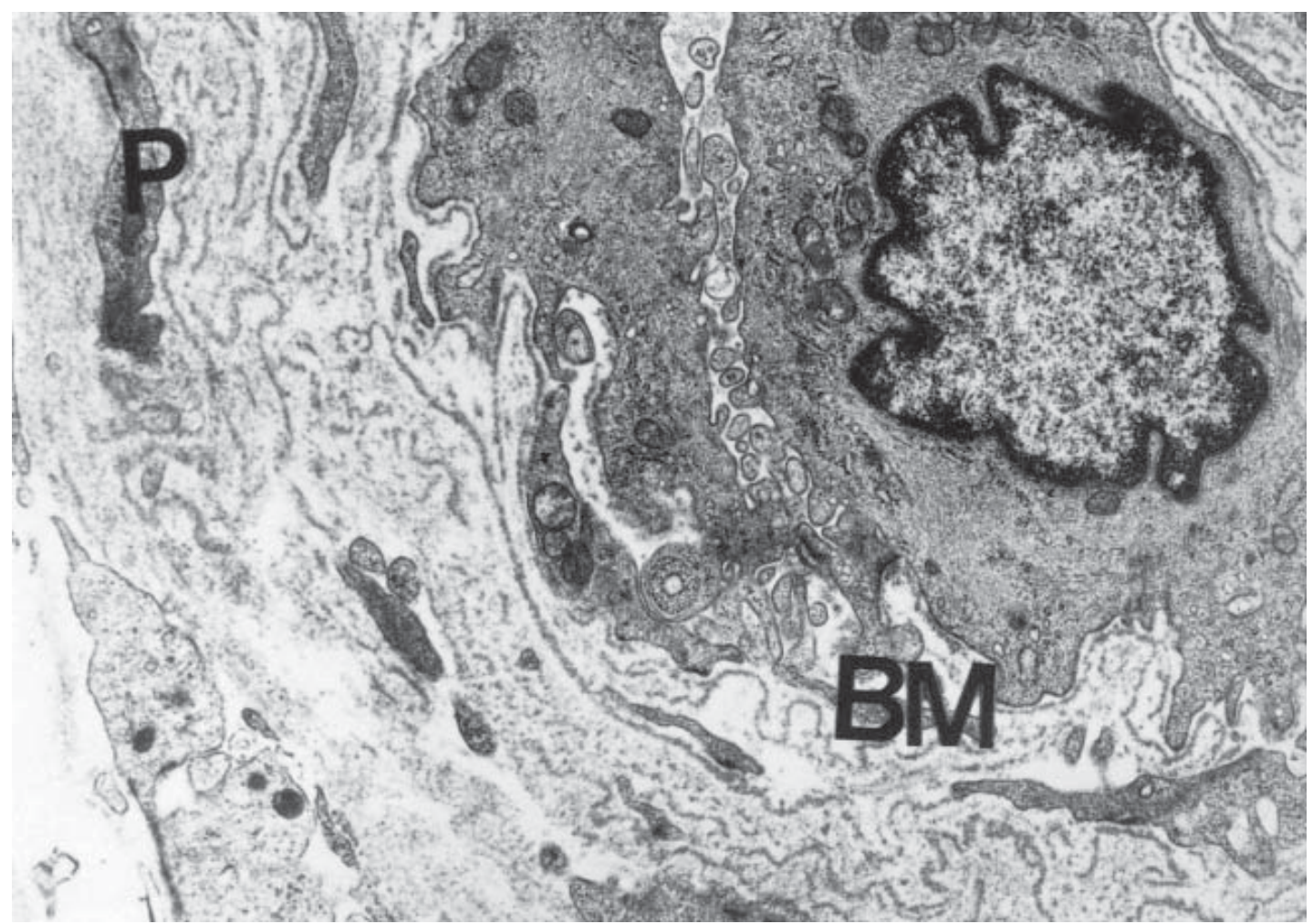

Figure 3. Vocal fold mucosa (TEM, magnification $\times 4,400)$. Fragment of the blood vessel with multiplication of the basal membrane (BM). Numerous branches of pericytes $(\mathrm{P})$

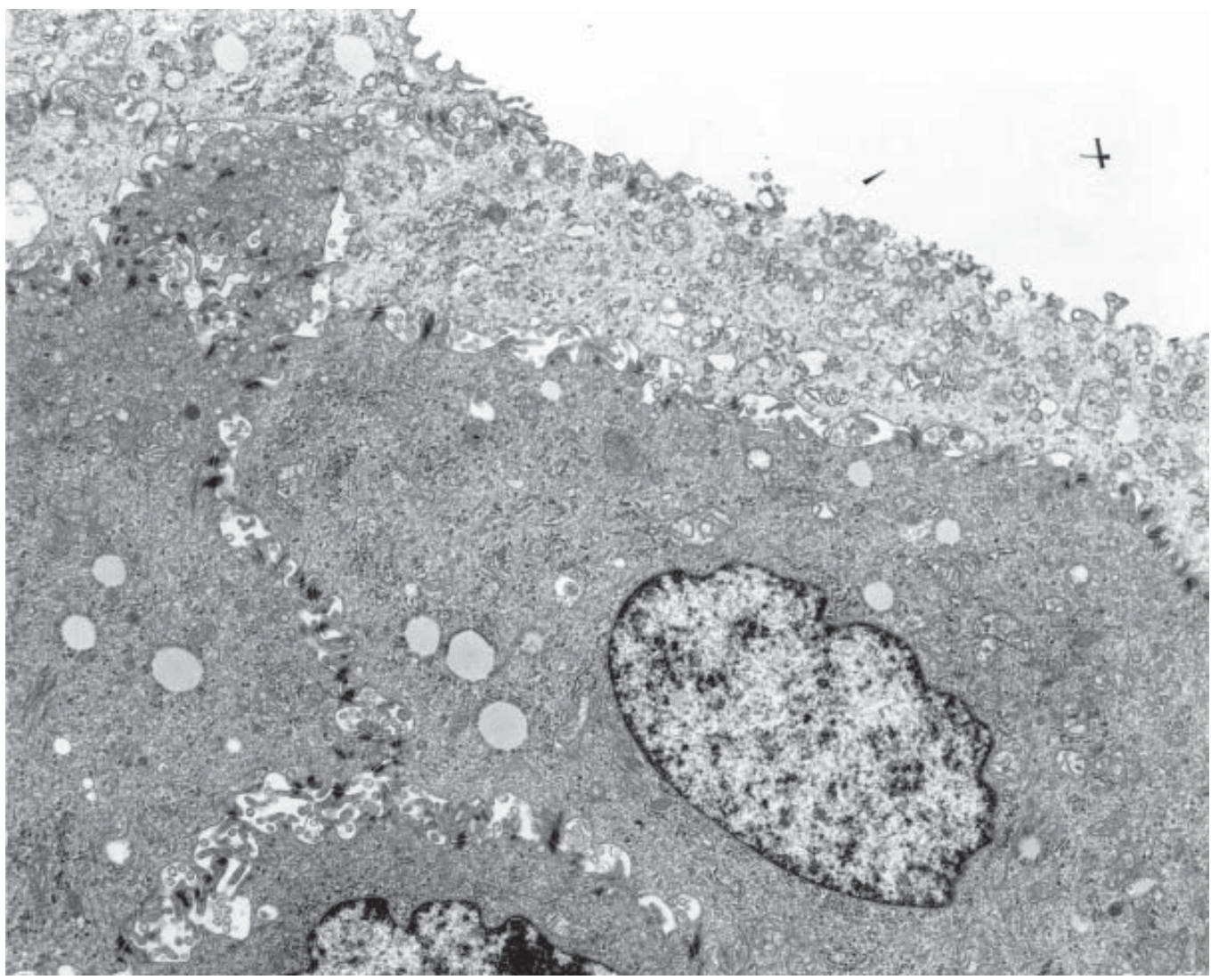

Figure 4. Epithelial cells of vocal fold $($ TEM, magnification $\times 3,000)$. Epithelial cells with intercellular spaces 


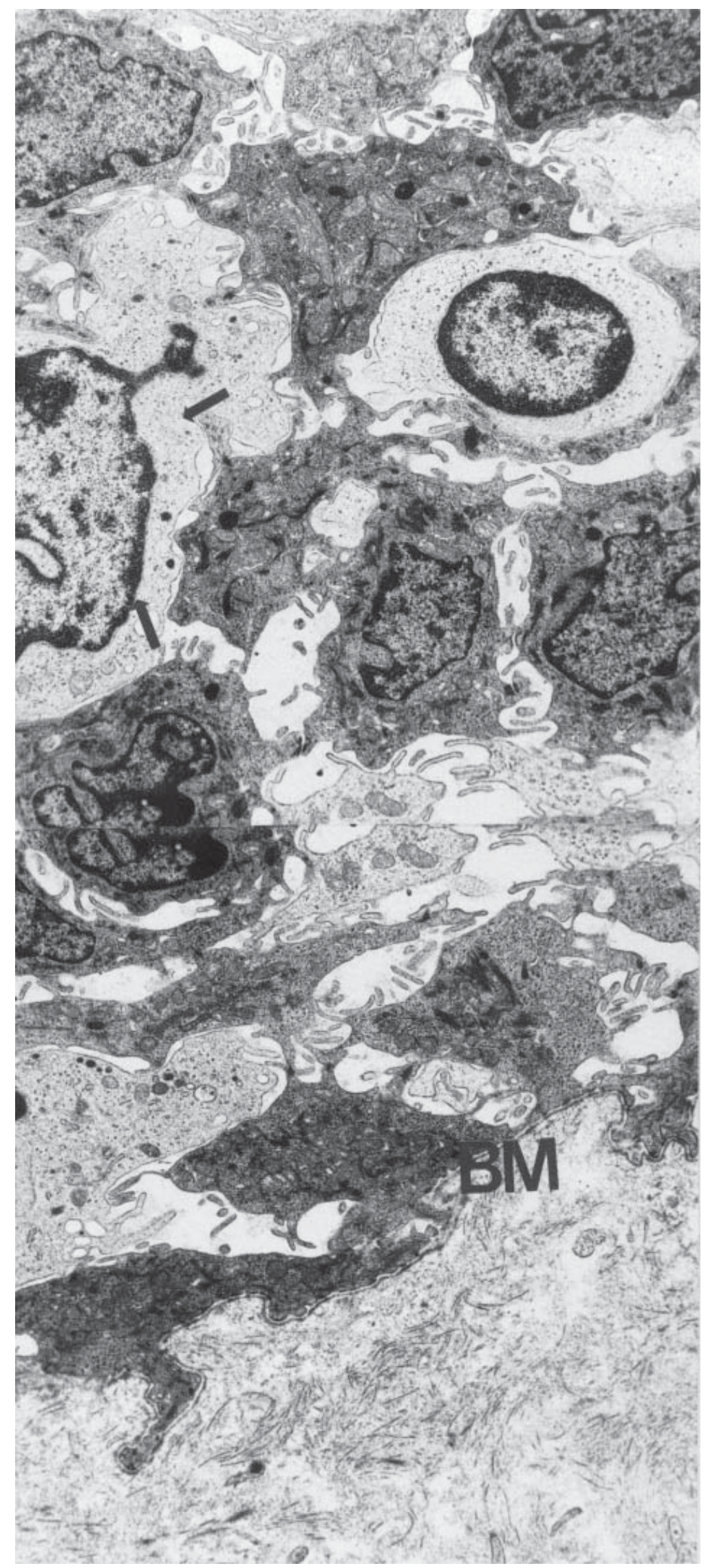

Figure 5. Layered structure of vocal fold mucosa (TEM $\times 3,000)$. Epithelial cells with intercellular spaces $(\uparrow)$. The basal membrane of epithelium with folds (BM). Scanty inflammatory cells in the epithelium and under the epithelium 


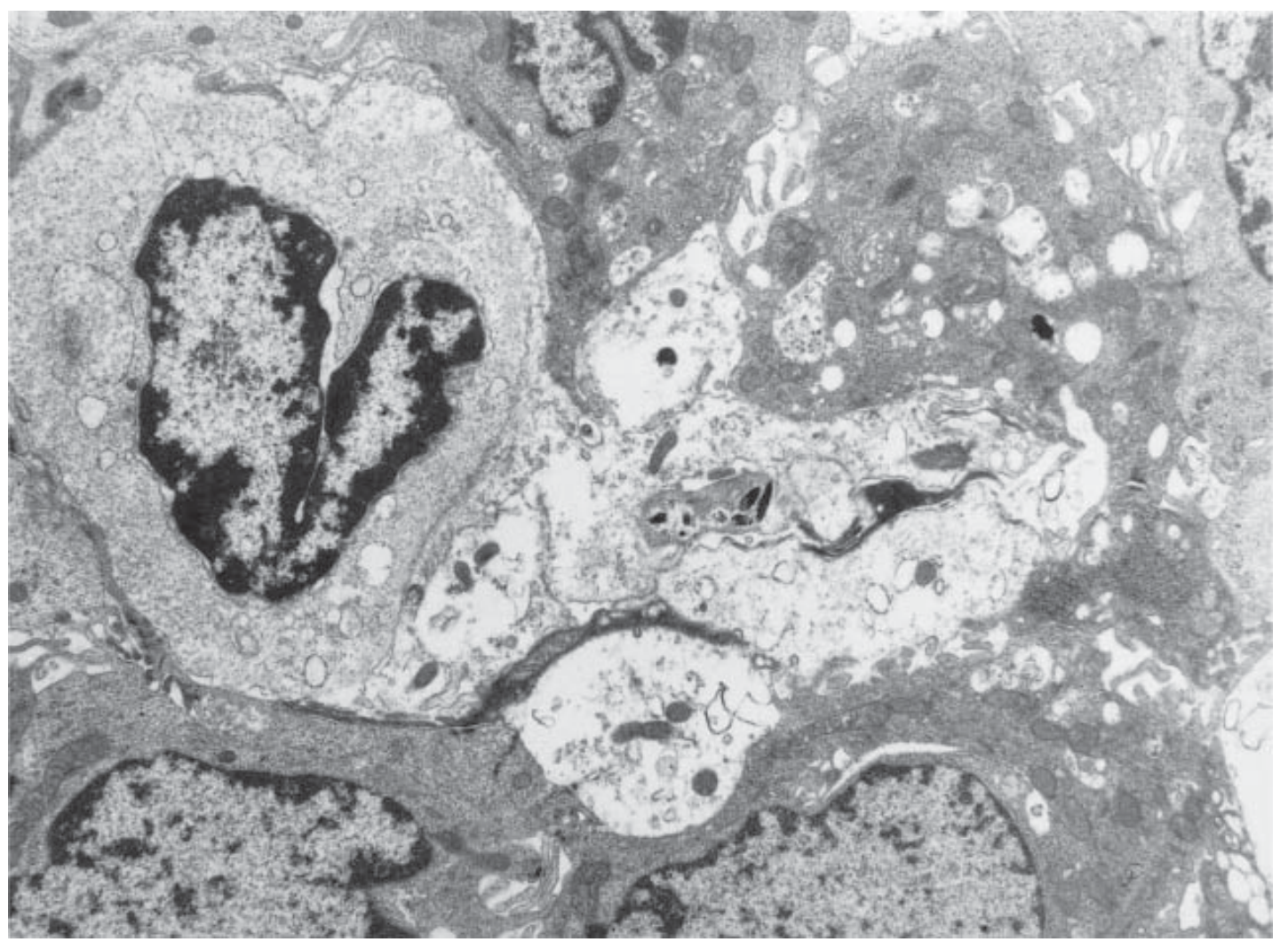

Figure 6. Epithelial cells with signs of vacuolization $(\mathrm{TEM} \times 3,000)$

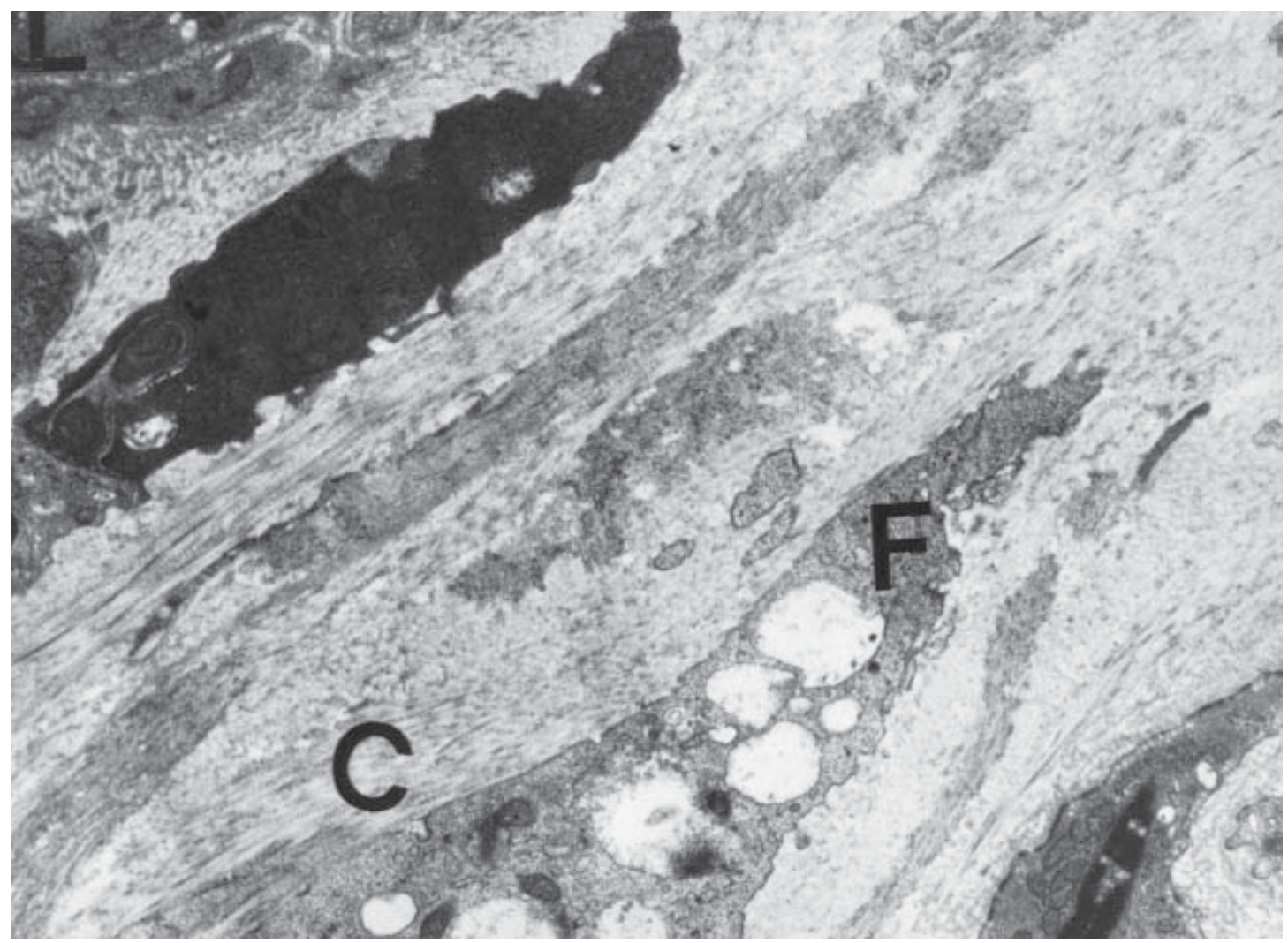

Figure 7. Vocal fold mucosa $($ TEM $\times 3,000)$. Collagenous fibers $(C)$ and fibroblasts $(F)$ with signs of vacuolization 


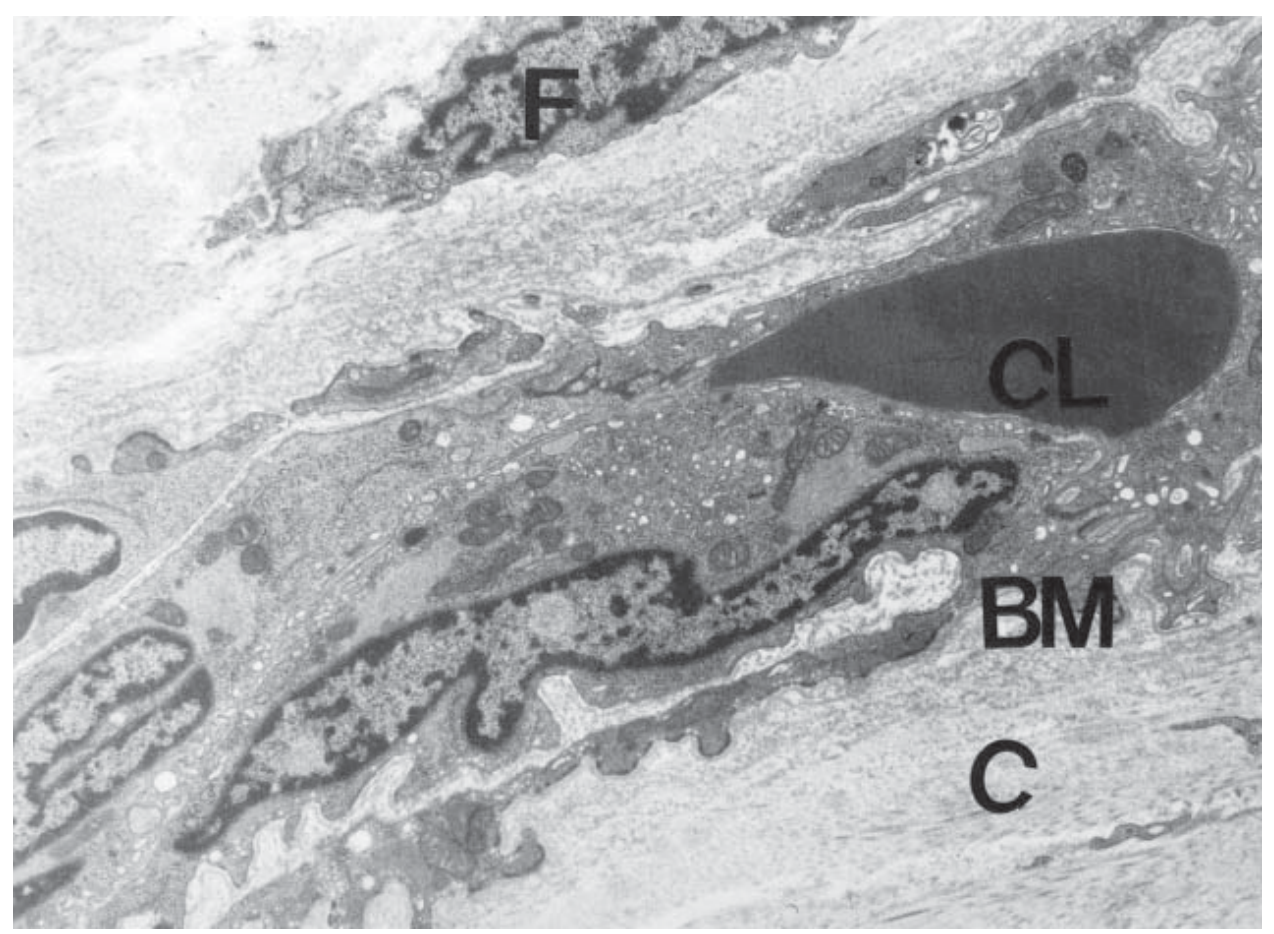

Figure 8. Vocal fold mucosa $(\mathrm{TEM} \times 3,000)$. Collagenous fibers $(\mathrm{C})$ and fibroblasts $(\mathrm{F})$. The blood vessel is visible $(\mathrm{CL})$. The basal membrane of epithelium with folds (BM)

2. Cells of the multilayered epithelium situated on the basal membrane with folds, numerous pericytes, scanty blood vessels and collagenous fibers determine the physiological vibration of the vocal folds.

3. In the analyzed groups of patients, the presence of small morphological changes suggesting polypoid degeneration or atrophy do not have a significant impact on voice quality in clinical assessment.

\section{References}

1. Sato K. Functional fine structures of the human vocal fold mucosa. In: Rubin JS, Sataloff RT, Korovin GS, ed. Diagnosis and treatment of voice disorders. San Diego, Oxford: Plural Publishing; 2006:47-72.

2. Kosztyła-Hojna B, Andrzejewska A, Rutkowski R, Rogowski M. Morphological aspect of voice disturbances of aged persons with coexisting hypopharynx cancer. Folia Histochem et Cytobiol. 2007;45:221-227.

3. Pruszewicz A. Laryngeal physiology. In: Pruszewicz A, ed. Clinical Phoniatrics. Warsaw: PZWL; 1992:36-50.

4. Gray SD, Hirano M, Sato K. Molecular and cellular structure of vocal fold tissue. In: Titze IR, ed. Vocal fold physiology. San Diego: Singular Publishing Group; 1993:1-35.

5. Tillmann E, Pietzsch-Rohrschneider I, Huenges H. The human vocal cord surface. Cell Tiss Res. 1977;185:279-283.

6. Hirano M, Koike Y, Hirose K, Kasuya T. Observation of mucous membrane of human vocal cords under electron microscopy. J Otolaryngol Jpn. 1974;77:650-660.

7. Sasaki CT, Kim Y, Hundal J. Anatomy of the human larynx. In: Rubin JS, Sataloff RT, Korovin GS, ed. Diagnosis and treatment of voice disorders. San Diego, Oxford: Plural Publishing; 2006:31-47.
8. Hirano M, Sato K, Nakashima T. Fibroblasts in human vocal fold mucosa. Acta Otolaryngol (Stockh.). 1999;119:271-276.

9. Hirano M, Sato K, Nakashima T. Fibroblasts in geriatric vocal fold mucosa. Acta Otolaryngol. 2000;120:336-340.

10. Sato K, Sakaguchi S, Kurita S, Hirano M. A morphological study of aged larynxes. Larynx Jpn. 1992;4:84-94.

11. Sato K, Hirano M. Age-related changes of elastic fibers in the superficial layer of the lamina propria of vocal folds. Ann Otol Rhinol Laryngol. 1997;106:44-48.

12. Sato K. Reticular fibers in the vocal fold mucosa. Ann Otol Rhinol Laryngol. 1998;107:1023-1028.

13. Sato K, Hirano M, Nakashima T. Stellate cells in the human vocal fold. Ann Otol Rhinol Laryngol. 2001;110:319-325.

14. Sato K, Hirano M, Nakashima T. Age-related changes of collagenous fibers in the human vocal folds mucosa. Ann Otol Rhinol Laryngol. 2002;111:15-20.

15. Zalesska-Kręcicka M, Kręcicki T, Jeleń M. Atlas of laryngeal diseases. Wrocław: Volumed; 1995.

16. Mihashi S, Okada M, Kurita S et al. Vascular network of the vocal fold. In: Stevens KN, Hirano M, ed. Vocal fold physiology. Tokyo: University of Tokyo press; 1981:45-59.

17. Sato K, Kashiragi S, Hirano M. Ultrastructure of the mucous membrane of the human newborn vocal folds. J Otolaryngol Jpn. 1997;100:479-483.

18. Sato K, Hirano M. Fine three-dimensional structure of pericytes in the vocal fold mucosa. Ann Otol Rhinol Laryngol. 1997;106:490-494.

19. Sato K, Hirano M, Nakashima T. Fine structure of the human newborn and infant vocal fold mucosa. Ann Otol Rhinol Laryngol. 2001;110:417-424.

Submitted: 14 July, 2010

Accepted after reviews: 30 January, 2011 